\title{
A comparative study of metagenomics analysis pipelines at the species level
}

Affiliations:

$4{ }^{1}$ Department of Molecular Biology, Cell Biology, and Biochemistry, Brown University,

5 Providence, RI 02912

$6 \quad{ }^{2}$ Center for Computational Molecular Biology, Brown University, Providence, RI 02912

7 Correspondence:

8 nicola_neretti@brown.edu (N. Neretti)

9 Abstract

Many metagenomics classification tools have been developed with the rapid growth of the metagenomics field. However, the classification of closely related species remains a challenge for this field. Here, we compared MetaPhlAn2, kallisto and Kraken for their performances in two metagenomics settings, human metagenomics and environmental metagenomics. Our comparative

14 study showed that kallisto demonstrated higher sensitivity than MetaPhlAn2 and Kraken and better quantification accuracy than Kraken at the species level. We also showed that classification tools that run on full reference genomes misidentified many species that were not truly present. In order to reduce false positives, we introduced marker genes from MetaPhlAn2 into our pipeline, which uses kallisto for the classification step, as an additional filtering step for species detection.

\section{Introduction}

21 The advent of shotgun metagenomic sequencing greatly facilitated the identification and

22 classification of microbes by providing a means to detect phenotypically aberrant or unculturable

23 microbes [1]. It allows a much faster and cheaper taxonomic profiling of microbial communities

24 in different ecosystems such as the microbiome in human, soil and ocean. With the rising use of 
shotgun metagenomic sequencing in the last decade, many microbial species classification tools have since been developed [2]. However, there still remains one of the main challenges in shotgun metagenomics analysis - the genomes similarity problem among closely related species in which

28 it is hard to distinguish and classify ambiguous reads. [3]. For instance, Shigella dysenteriae and Escherichia coli that share relatively similar genomes complicate the taxonomic assignment at the genus, species and strain levels [4]. Several tools such as MetaPhlAn and MetaPhyler have been developed to profile microbial communities rapidly using a set of marker sequences. The use of markers can reduce ambiguous reads mapping to multiple genomes [5-8], but at the same time, not all sequencing reads can be classified. This poses a limit to perform a detailed analysis on the samples, such as gene content estimations [9]. Other tools such as Kraken [9] and Clark [10] also have been developed for high accuracy microbial sequence classification. These tools represent read-alignment free and k-mer based approach that can classify sequencing reads accurately and rapidly. Lindgreen et al. evaluated many of the widely used metagenomics classification tools and the comparison of the overall performances showed that Kraken performs best in terms of the speed and accuracy in identifying taxonomic distribution [11]. Interestingly, an RNA-seq quantification tool, kallisto, has also been tested and compared to Kraken in the metagenomics setting. Kallisto is a fast k-mer based pseudoalignment approach of RNA-seq reads to quantify

42 isoform expression level using a transcriptome De Bruijn graph (T-DBG) method [12]. The comparison of Kraken and kallisto by Schaeffer et al. showed that kallisto outperforms Kraken in

44 the metagenomics quantification at three taxonomic ranks: genus, species and strain [13]. However, have been reported in the study. 
48 Here, we compared and evaluated different combinations of tools, including MetaPhlAn2, kallisto and Kraken, in terms of their detection and quantification performances, speed and memory requirement in microbial species level classification. We showed that the performance of kallisto

51 is better than Kraken in terms of the quantification at the species level, which is consistent with

52 the result showed by Schaeffer et al [13]. However, while kallisto uses expectation maximization

53 (EM) algorithm to probabilistically handle ambiguous reads, the FDR still increases dramatically

54 as the number of sequencing reads increases. Therefore, we incorporated a collection of species-

55 specific markers genes from MetaPhlAn2 into the kallisto quantification pipeline as an additional

56 step to reduce the FDR. In addition, due to the very high memory requirement to build the T-DBG

57 by kallisto, we also introduced another pipeline that build the index only on detected species from

58 the kallisto run on marker genes to evaluate the performance of kallisto at a larger scale of 59 reference genomes. Overall, we showed that the kallisto run on full microbial genome alone is not 60 sufficient because it detected many other species that were not there. The use of marker genes is necessary to reduce the high false positives.

\section{Results}

64 Two different microbial community samples were simulated: (i) human-associated habitat 65 microbial community samples that consist of 5\% microbial reads, and (ii) samples that consist of only microbial reads. We tested five pipelines that use either MetaPhlan2, Kraken or kallisto

67 (Figure 9 and Figure 10) for the classification of reads at the species level and evaluated their 68 performances in terms of sensitivity, FDR, rate of false negative and memory requirement. 
71 For datasets that mimic samples extracted from human-associated habitat, we first filtered out

72 human reads before running the classification step. Sensitivity measures how well the pipelines

73 detect species that were truly present in the samples. Pipelines that include kallisto (Figure 9 B1-

74 D1) in the classification step demonstrated the highest sensitivity, followed by Kraken (Figure 9

75 E1) and MetaPhlAn2 (Figure 9 A1, Figure 1a). MetaPhlAn2 performed poorly at low number of

76 microbial reads due to the use of only marker genes and not full genomes. FDR measures the

77 number of species that were detected but not in the samples. It increases dramatically as the number

78 of reads increases when full microbial genomes were used in the reference database (Figure 1b).

79 In contrast, pipelines that involve additional filtering step using species-specific marker genes from MetaPhlAn2 (C1 and D1) and MetaPhlAn2 itself (A1) demonstrated lower FDR (<0.05) compared

81 to the pipelines that only classify reads using full microbial genomes. Rate of false negative refers

82 to the frequency of not detecting species that were present in the samples. Pipelines that include

83 kallisto (B1-D1) showed the lowest rate of false negative, followed by Kraken (E1) and

84 MetaPhlAn2 (A1) (Figure 1c). Although kallisto performed well in the detection of species, the

85 downside of it is that it requires high memory in the index and quantification steps. Specifically,

86 kallisto quantification (B1 and C1) with a database that consists of 3511 fasta sequences ( 5.6GB)

87 consumed approximately 300 GB of memory (Figure 1d). Therefore, out of the five pipelines that

88 we tested, we included one that index only genomes that were detected in the marker genes

89 detection step (D1), in order to reduce the size of the database used in the final quantification. In

90 this case, the memory requirement will vary depending on the number of species detected. The

91 number of genomes in our simulated dataset was 315 and kallisto required approximately 38GB

92 of memory for the quantification step. In addition, the run time also varies between different

93 pipelines (Figure 2a-e). On a 16-core server, MetaPhlAn2 demonstrated the shortest run time due 
94 to its small database that consist of only marker genes. Kraken pipeline had the second shortest

95 run time, followed by the three kallisto pipelines, with $\mathrm{B} 1$ and $\mathrm{C} 1$ that run on the full microbial

96 genome having the longest run time. Similar to the memory requirement by the D1 pipeline, the

97 run time that involves building a kallisto index and running on a reduced database will vary

98 depending on the number of species detected.

a
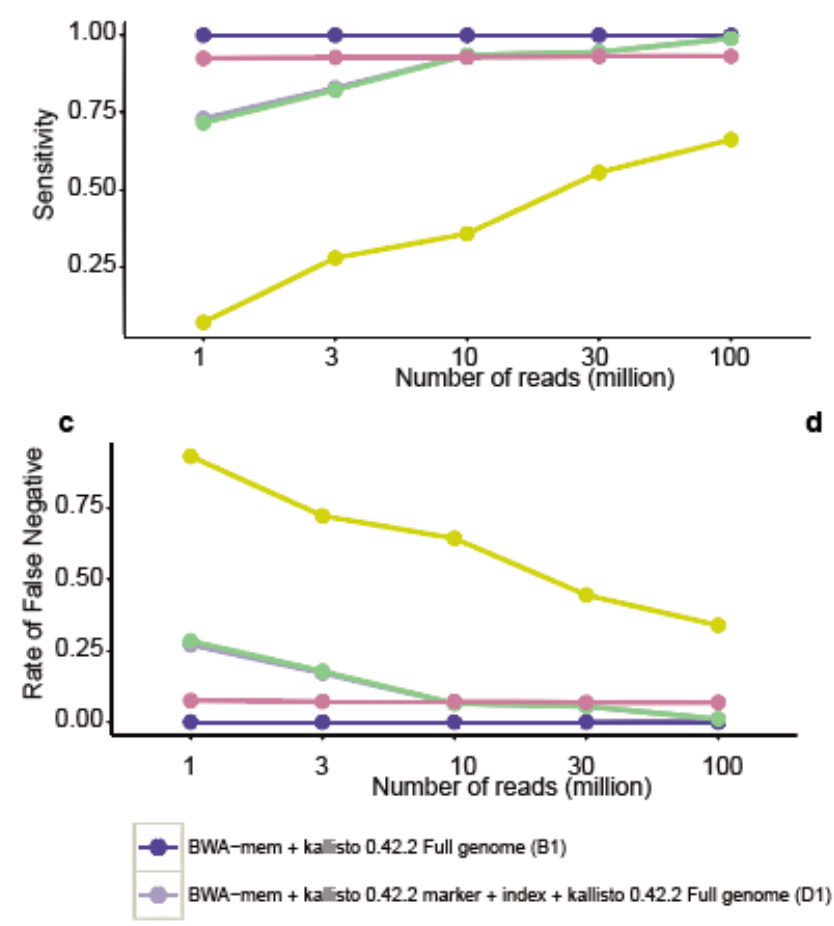
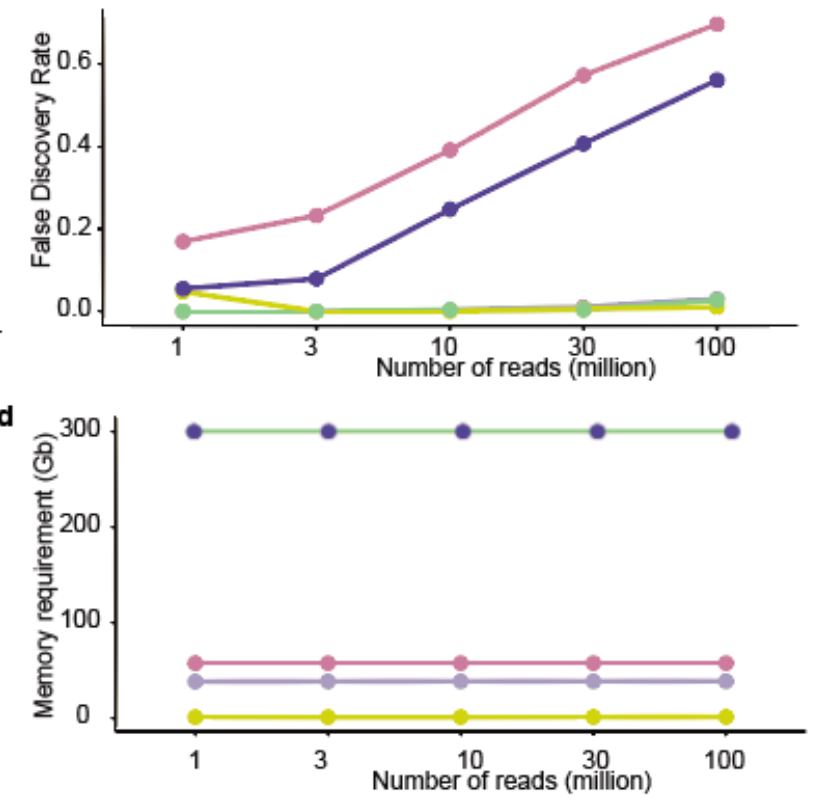
BWA-mem + kall sto 0.42 .2 marker
kallisto 0.42 .2 Full genome (C1)

Figure 1: (a) Sensitivity, (b) false discovery rate, (c) rate of false negative and (d) memory requirement for simulated microbial reads extracted from human-associated habitat are used in the estimation of microbial composition. The kallisto pipeline (D1) that has a filtration step with species-specific markers demonstrated the highest accuracy in estimating species counts as the number of reads increases, followed by the other two kallisto pipelines (C1 and B1), and 
111 database based on detected species.
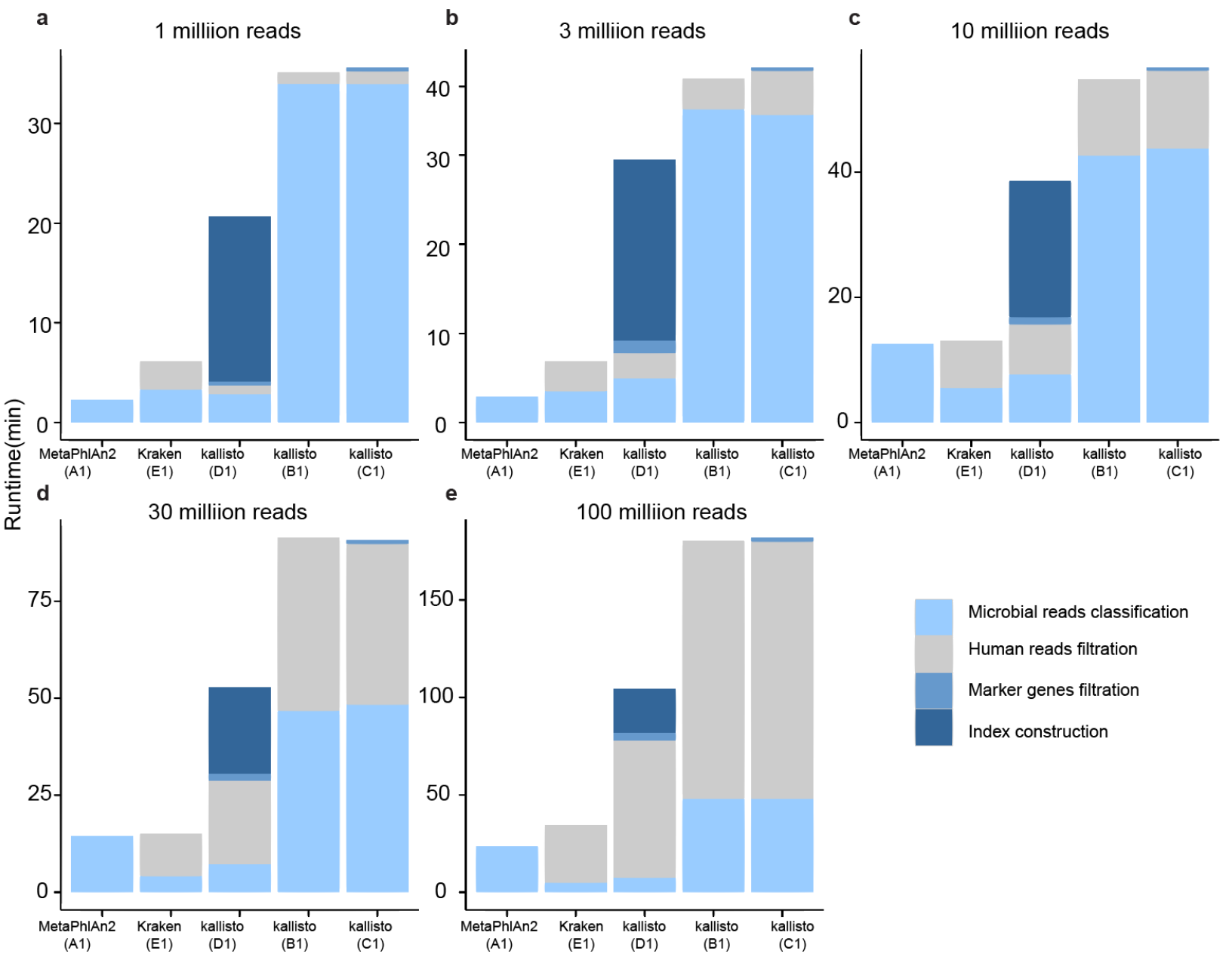

113 Figure 2 Runtime ( $\mathrm{min}$ ) for samples extracted from human-associated habitat that consist of (a) 1

114 million, (b) 3 million, (c) 10 million, (d) 30 million, and (e) 100 million total reads 
117 The same five pipelines used in the first part of the study minus the human filtration step were

118 evaluated for their performances in classifying samples with only microbial reads. The

119 performances of each pipeline were similar to the first part of the study (Figure 3a-d), in which

120 pipelines that include kallisto (Figure 10 B2-D2) in the classification step demonstrated the highest

121 sensitivity, followed by Kraken (Figure 10 E2) and MetaPhlAn2 (Figure 10 A2). Pipelines that do

122 not have a filtering step using species-specific marker genes (B2 and E2) demonstrated higher

123 FDR as the number of reads increases. Kallisto pipelines (B2 and C2) are not practical because it

124 requires memory as high as $300 \mathrm{~GB}$ for a database of only 3511 microbial reference sequences

125 (Figure 3d).
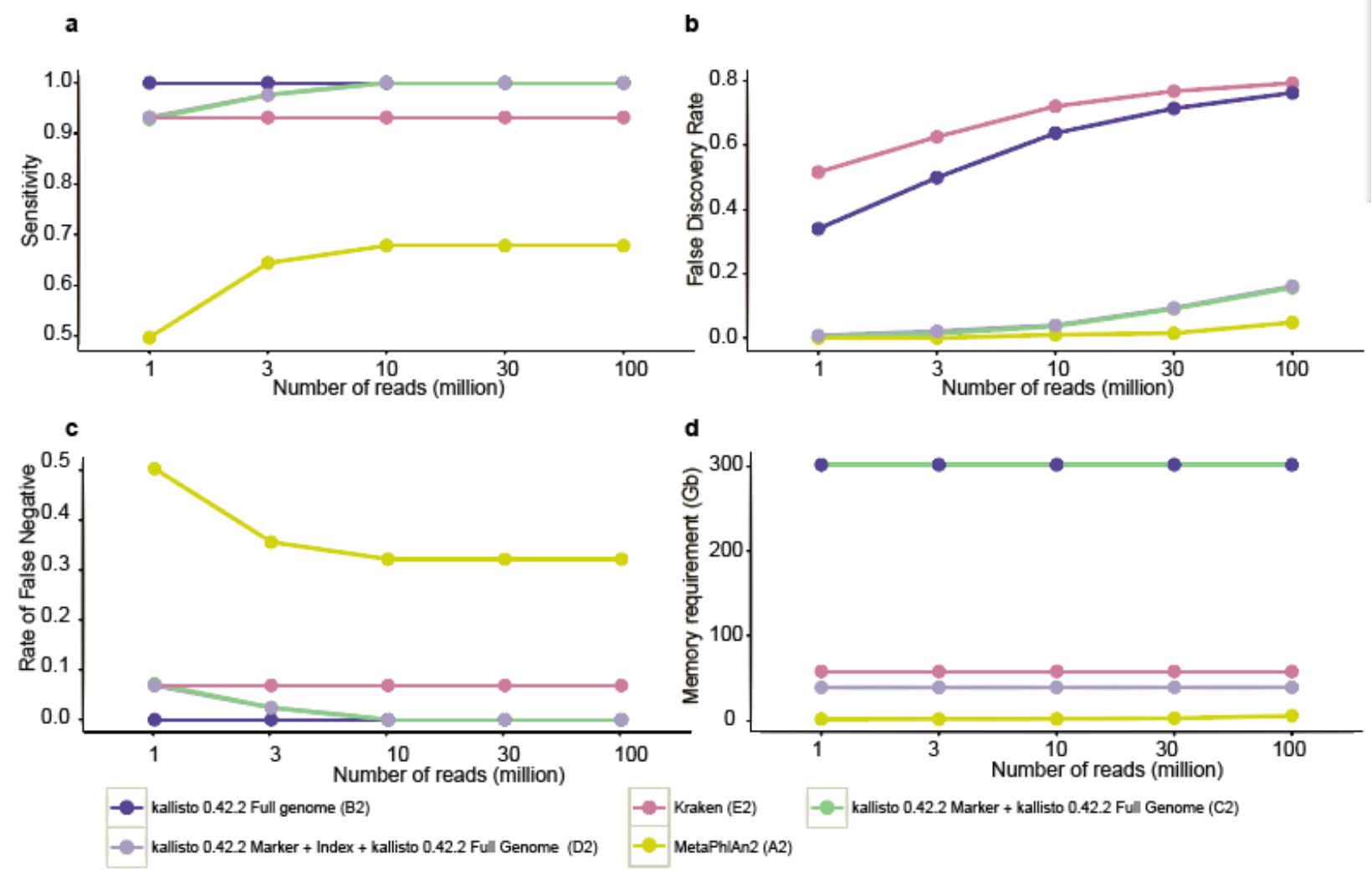

127 Figure 3: (a) Sensitivity, (b) false discovery rate, (c) rate of false negative and (d) memory requirement for simulated samples that contain only microbial reads 
130 In terms of the speed, MetaPhlAn2 (A2) had the shortest run time on a 16-core server. However,

131 as the number of reads increases, Kraken (E2) outperformed MetaPhlAn2 in terms of the run time

132 (Figure 4a-e) even though it runs on a full reference genome database. Kraken could classify 100

133 million reads in about 30 minutes. Kallisto (B2 and C2) had the longest run time for microbial

134 reads classification when full reference genomes database was used.

135 The kallisto pipeline (B2) without the filtration step with species-specific markers demonstrated

136 the highest accuracy in estimating species counts, followed by the other two kallisto pipelines (D2

137 and C2), and then Kraken (E2). The downside of this B2 pipeline was that the FDR increases

138 dramatically to as high as 0.76 with 100 million of microbial sequencing reads (Figure $3 b$ ). This

139 indicates that kallisto quantification alone is not sufficient to accurately identify the species that

140 are present in a sample and the use of marker genes is necessary to reduce the FDR. The additional

141 marker genes filtration step will definitely require more run time than without one. The

142 quantification step by kallisto also consumes a much longer time (10 times as long when

143 quantifying 1 million microbial reads) when compared to Kraken (Figure 4a). Overall, in order to

144 get an accurate quantification of microbial reads, yet the highest sensitivity and the lowest FDR,

145 the D2 pipeline demonstrated the most optimal combination of all parameters tested, although the

146 run time is not the best. The first step is the species level detection using marker genes to reduce

147 the FDR, followed by a quantification step on a selected full genome database based on detected

148 species. 
bioRxiv preprint doi: https://doi.org/10.1101/081141; this version posted October 15,2016 . The copyright holder for this preprint (which was not certified by peer review) is the author/funder, who has granted bioRxiv a license to display the preprint in perpetuity. It is made available under aCC-BY-NC-ND 4.0 International license.
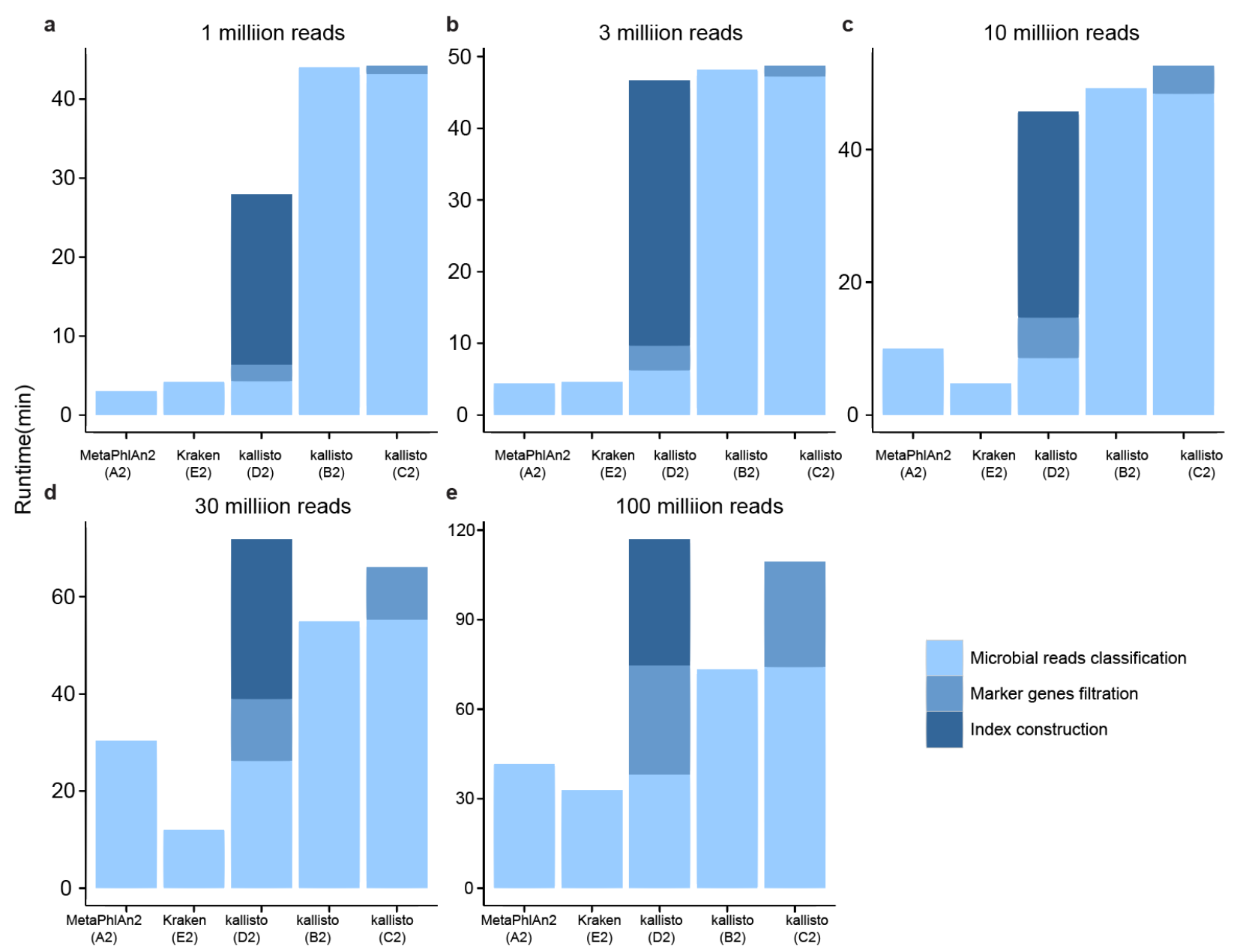

150 Figure 4: Runtime (min) for simulated samples that consist of (a) 1 million, (b) 3 million, (c) 10

151 million, (d) 30 million, and (e) 100 million microbial reads 

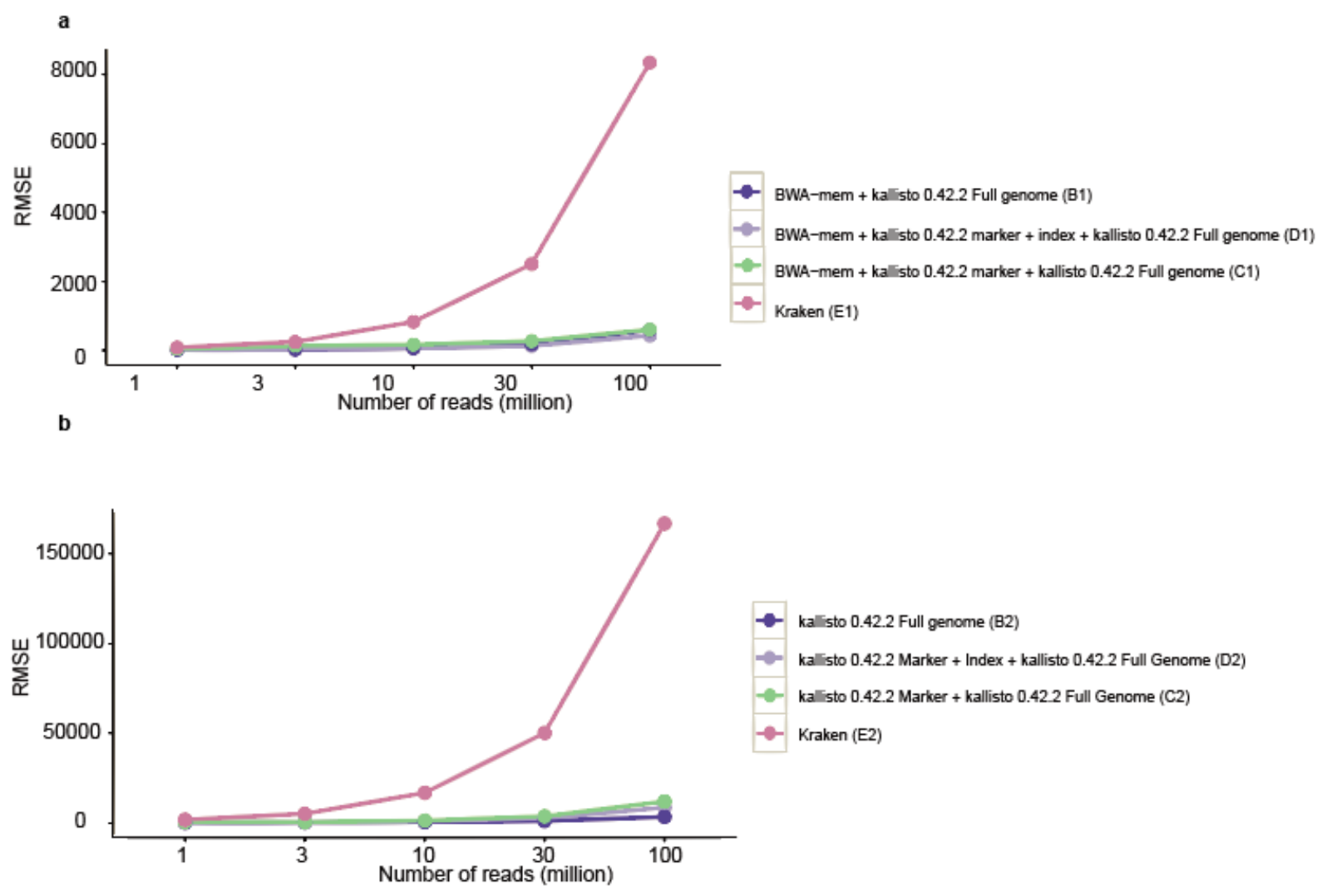

153

154 Figure 5: RMSE of 292 organisms (a) from human-associated habitat and (b) of all microbial

155 reads only, at the species level

In addition to our simulated sequencing reads, we also tested the D2 pipeline on the dataset that was used by Schaeffer et al. to test kallisto in a metagenomics setting. $56.9 \%$ of the reads are from strains found in our microbial reference genomes database, $34.1 \%$ of them are of other strains that

161 can be classified into species found in the database, and the remaining $9 \%$ are not in any of the 162 species found in the database. We wanted to test how well the D2 pipeline can classify when some 
163 of the reads are not found in the database because this can mimic the problem in analyzing real

164 dataset. The pipeline correctly identified 67 species out of the 85 species that were truly present in

165 the dataset and misidentified 72 species. The quantification accuracy of this approach was shown

166 in Figure $6($ Pearson $r=0.75)$.

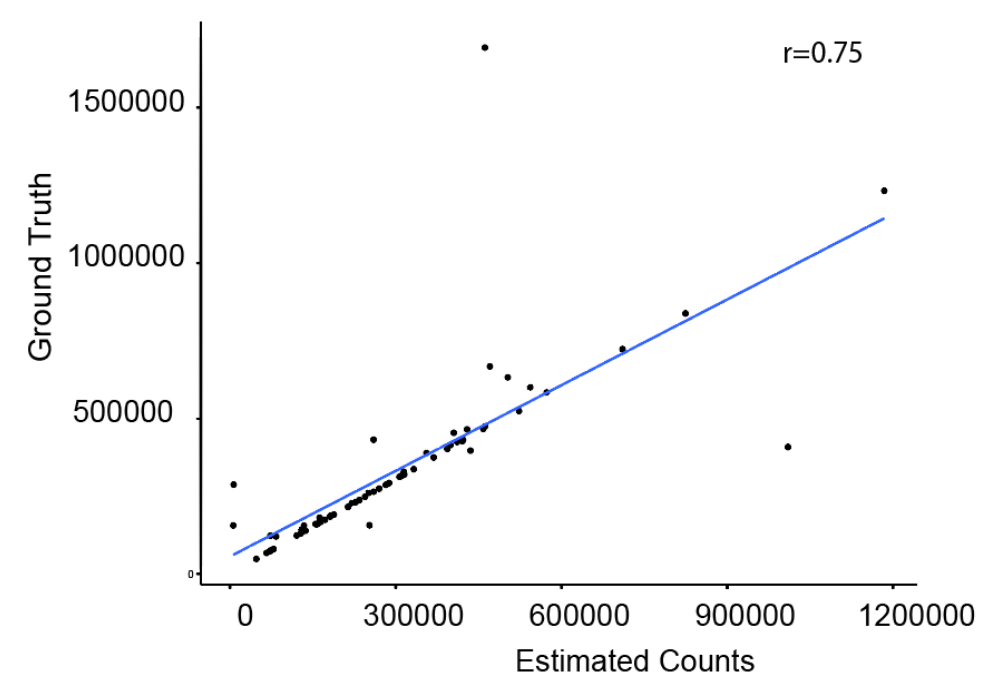

168 Figure 6: Quantification accuracy for Illumina100 dataset using the D2 pipeline

169 (iv) Stool and saliva sequence data from the HPFS cohort 

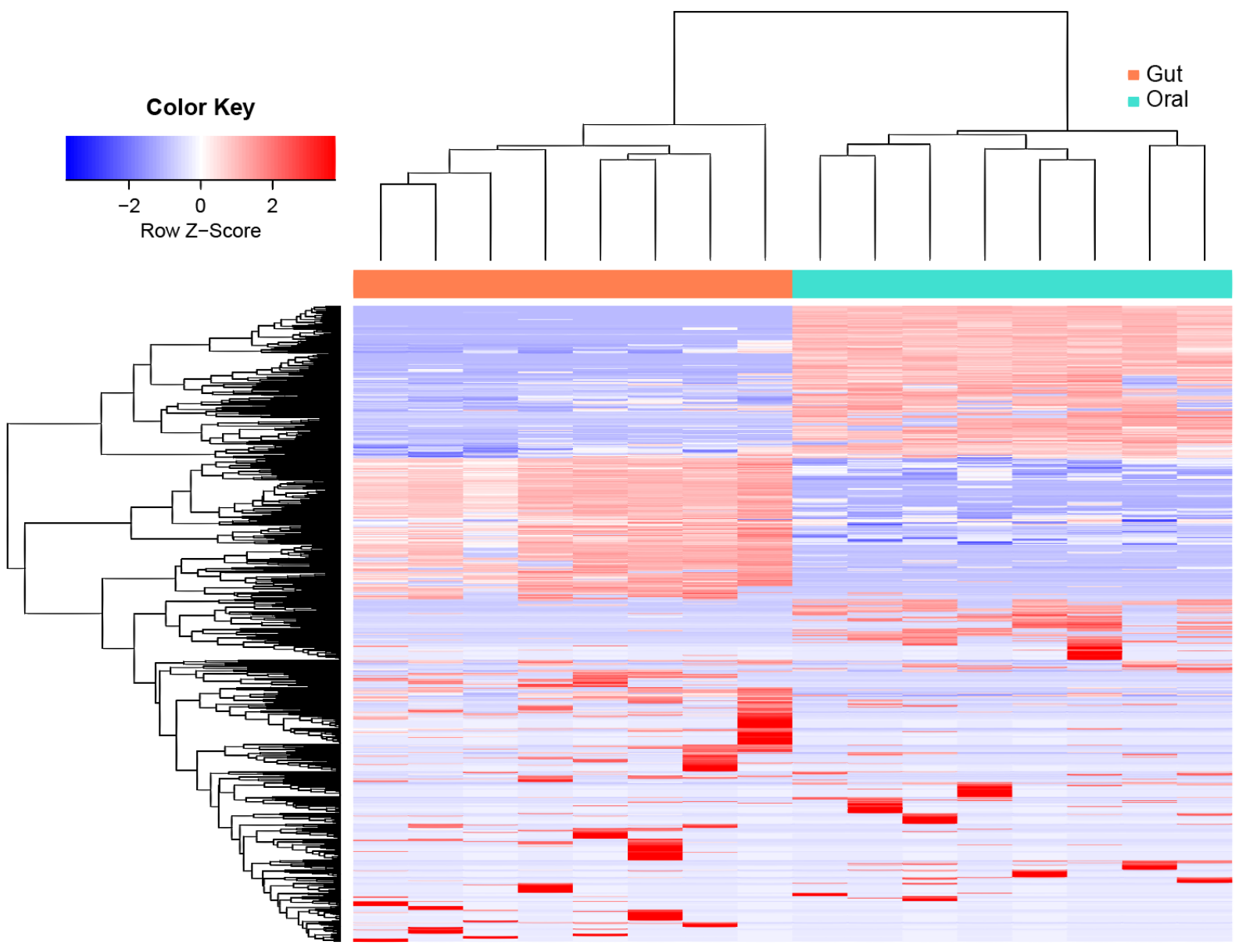

171 Figure 7: Gut and oral microbiome from the HPFS cohort.

172 We obtained stool and saliva samples from eight healthy Health Professionals Follow-Up Study

173 (HPFS) subjects and analyzed using the D1 pipeline. There was a clear clustering structure (Figure

1747 ) between the oral and the gut microbiome of the eight HPFS subjects. The most distinctive

175 species composition that distinguish between the oral and the gut microbiome were members from

176 the Prevotella genus and the Bacteroides genus (Figure 8a). Members in the Prevotella genus such

177 as Prevotella histicola, Prevotella melaninogenica, Prevotella pallens and Prevotella sp. C561

178 were found in all eight oral samples but not in any of the gut sample (FDR $\left.<3.6 \times 10^{-16}\right)$. In contrast,

179 species in the Bacteroides genus such as Bacteroides sp. 91 42FAA, Bacteroides sp. HPSO048, 
181 found in the gut samples (FDR $\left.<3.1 \times 10^{-5}\right)$. In addition, we also identified two species, Veilonella sp. HPA0037 and Streptococcus salivarius with at least 1000 counts per million (CPM), in both the gut and the oral samples from the same individual, in at least two of them. The link between the oral and the gut microbiome from the same dataset has previously been shown in [14] and our finding on Streptococcus salivarius and the Veilonella genus was consistent with their result.
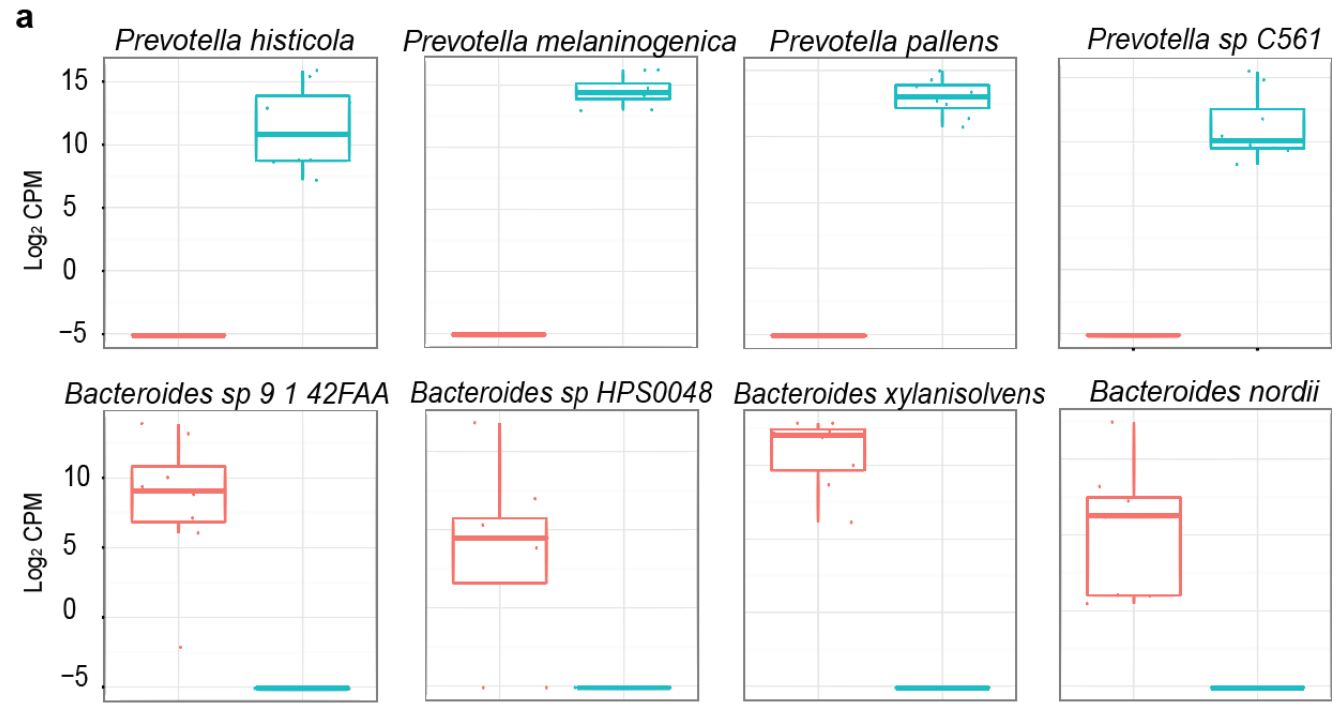

b
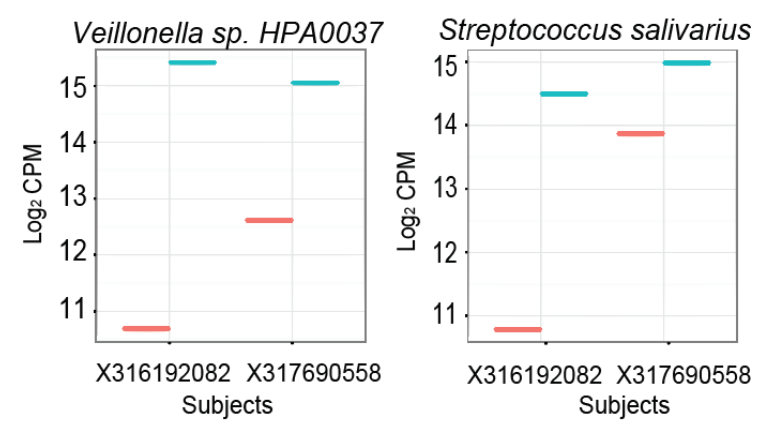

Figure 8: (a) Comparison of the species composition in gut and oral samples. The largest distinction between the oral and the gut microbiome were from several members in the Prevotella and Bacteroides genus. (b) Link between the oral and the gut microbiome. Two species with > $1000 \mathrm{CPM}$ were found in the oral and the gut samples from the same individual (of at least two individuals): Veillonella sp. HPA0037 and Streptococcus salivarius. 


\section{Discussion}

193
The classification of homologous sequences from closely related species can be challenging in shotgun metagenomics sequencing analysis because this can lead to many misidentifications of species. In addition, samples deriving from a host may contain many contaminating host DNA, thus complicating the data analysis [15]. In recent years, many metagenomic reads classification tools have been developed with the rising use of shogun metagenomics sequencing. While most of the studies have been focusing on bacterial communities, there is also a rising use of shotgun sequencing in viral community setting [16]. In this study, we tested different pipelines with existing metagenomics and RNA-seq data analysis tools on two different microbial communities, one that mimics samples deriving from human-associated habitat, and another one that consists of only microbial reads.

In the pipelines involving the use of kallisto for quantification, we first filter out human reads using BWA-MEM. The reason why we did not use kallisto for the human filtration step was because the index could not be built from the hg19 genome due to memory issue, as kallisto is specifically designed for transcriptome. With the same set of marker genes as the reference database, kallisto was shown to outperform MetaPhlAn2 in identifying the correct species. We introduced this marker genes filtration approach in our pipeline as a species detection step to reduce false positives. We showed that without this step, many species that are not truly present were detected. However, the drawback of this pipeline is the runtime. Kallisto is a fast pseudoaligner for transcriptome [12], but it takes a much longer time for metagenome sequence classification when compared to Kraken, an ultrafast metagenomics analysis tool. However, kallisto outperformed Kraken in terms of quantification accuracy. Overall, D1 (with human filtration) and D2 pipelines demonstrated the most optimal combinations of the performance parameters that we tested. 
215 To further test the pipelines, we applied the D2 method on iMESSi simulated DNA sequencing

216 reads. Due to the absence of reference genomes to half of the reads present in the simulated sample,

217 the sensitivity was not ideal. This can mimic the problem in analyzing real dataset in which there

218 is still a problem in classifying reads extracted from unknown species. In addition, many species

219 under the same genus were misidentified, including the Bacillus genus, the Thermus genus and the

220 Burkholderia genus. This suggests that some of the markers were not truly unique to the species

221 even after filtering out quasi-markers from MetaPhlAn2.

222 We also tested the D1 pipeline on real metagenomics sequence reads from the HPFS cohort (eight

223 healthy males at the Boston area). We showed that the highest distinction between the gut and the

224 oral samples came from members of two genus, Bacteroides and Prevotella. Several members in

225 the Bacteroides genus was found in the gut but not in the oral samples and vice versa for the

226 Prevotella genus. This is in agreement with previous result indicating that Bacteroides species in

227 the gut microbiome is associated with an animal-protein based modern western diet whereas

228 Prevotella species in the gut is associated with a carbohydrate-based rural diet [17]. We also found

229 several Prevotella species that were found only in the oral site but not in the gut samples. This is

230 consistent with the result in which Prevotella genus was previously shown to significantly

231 associate with the tongue dorsum [18].

\section{Methods}

\section{Generation of simulated DNA sequencing data}

234 We simulated DNA sequencing paired-end reads to represent two different microbial communities:

235 (i) microbial reads extracted from human host (consist of human reads and $\sim 5 \%$ of microbial reads)

236 and (ii) reads that consist of only microbial reads. To generate these reads, we used wgsim by 
237 Heng Li (https://github.com/lh3/wgsim) with hg19 genome and 315 microbial genomes (292

238 species: 74 bacteriophages, 69 viruses and 149 bacteria) from the NCBI RefSeq database [19]. We

239 used the default options in wgsim except for a rate of mutation of 0.003 , for the generation of

240 microbial reads. For the simulation that contains microbial reads from human host, we generated

241 five sets of them progressively, with approximately a total of 100 million reads $(94,999,998$ human

242 reads $+5,000,001$ microbial reads $), 30$ million reads $(28,500,000$ human reads $+1,499,999$

243 microbial reads), 10 million reads $(9,500,000$ human reads $+499,993$ microbial reads $), 3$ million

244 reads $(2,849,998$ human reads $+149,987$ microbial reads) and 1 million reads (949,997 human

245 reads $+49,997$ microbial reads). For the simulation that contains only microbial reads, we also

246 generated five sets of reads progressively, which contain approximately 100 million reads

$247(99,999,999), 30$ million reads $(30,000,001), 10$ million reads $(10,000,003), 3$ million reads

$248(2,999,998)$ and 1 million reads $(999,994)$.

\section{IMESS_Illumina simulated Illumina100}

250 In addition to our simulated sequencing reads, we also evaluated the D2 approach on the dataset

251 that was used by Schaeffer et al. to test kallisto in a metagenomics setting [13]. This dataset was

252 simulated using iMESS_Illumina and contains 100 bacterial genomes [20].

\section{Quantification of microbial reads}

254 We compared MetaPhlAn2, kallisto/0.42.2 and kraken/0.10.5-beta for their performance in 255 microbial reads quantification. For the simulated microbial sample of human origin, we first 256 filtered out reads mapping to hg19 genome [21], with the exception when using MetaPhlAn2 for 257 classification. No prior filtration is required for MetaPhlAn2 due to the use of microbial species258 specific markers database. For the approach involving Kraken (E1), we directly used Kraken to 
259 filter out human reads with the options, --quick, -- min-hits 5 --unclassified-out, with hg19 genome

260 as the reference genome. Next, we ran Kraken on the unclassified reads output against the full

261 microbial reference genomes database. For the approaches using kallisto, we compared three

262 different ways (B1-D1) to classify microbial reads. In the first approach (B1), we ran BWA-MEM

263 [22] against hg19 genome to filter out human reads. Then, we classify unmapped reads with the

264 full microbial reference genomes database using kallisto. In the second approach (C1), we ran an 265 additional step of kallisto on hg19 unmapped reads with species-specific markers from 266 MetaPhlAn2 as the reference genomes. This step is introduced to reduce the FDR and it is used to 267 detect the presence or the absence of a species, and not for quantification purpose. If a species is 268 detected here, the estimated read count will be obtained from the earlier step that uses full 269 microbial genomes database. In the third kallisto approach (D1), we again ran BWA-MEM against 270 hg19 genome for human reads filtration. The unmapped reads were classified using kallisto, with 271 species-specific markers from MetaPhlAn2 as the reference genomes to detect the presence of a 272 species. After identifying species that were present in the sample, we proceeded to build a kallisto 273 index on full microbial genomes only on these selected species. The quantification step was ran 274 with this reduced database. 


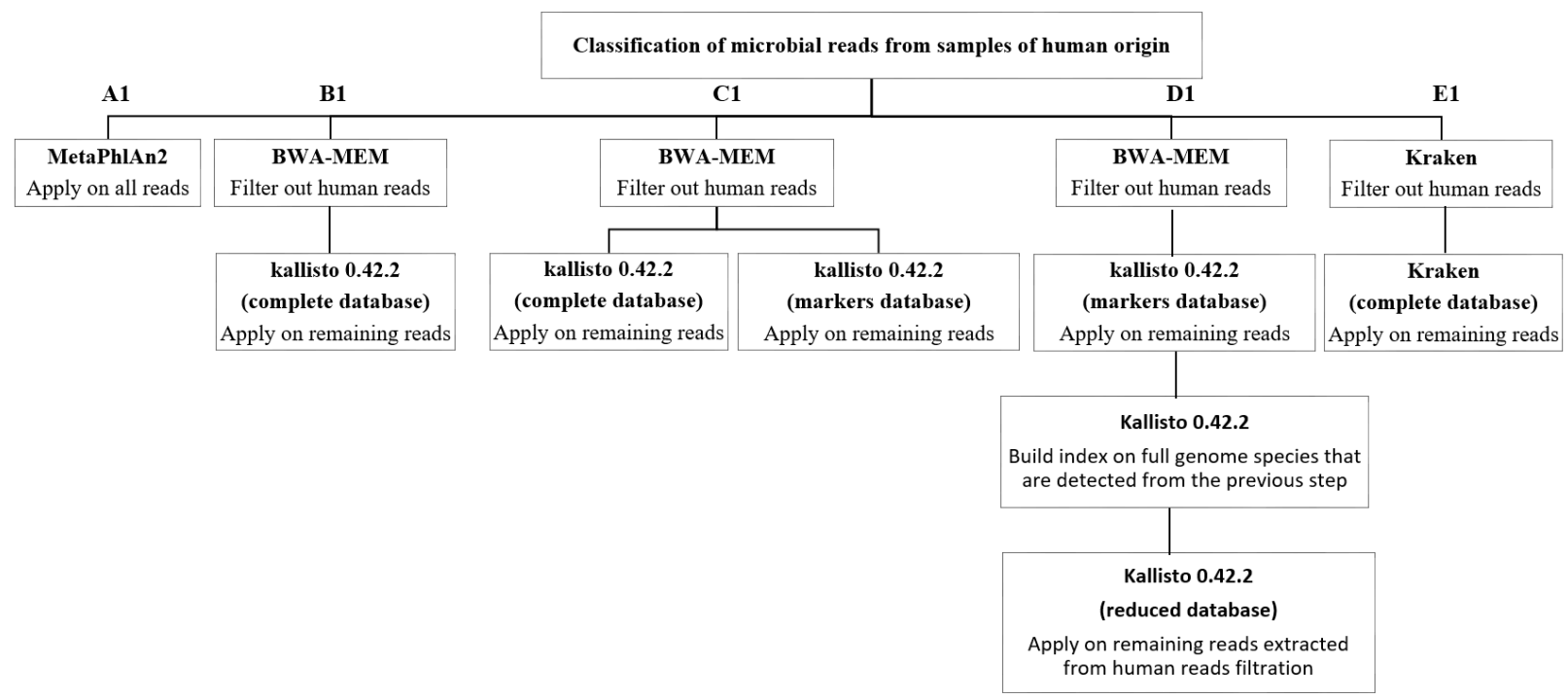

Figure 9: Flowchart of analysis pipelines for samples extracted from human-associated habitat.

277 Five pipelines (A1-E1) were evaluated and compared for their overall performances.

278 For the second microbial community that contains only microbial reads, we ran MetaPhlAn2,

279 kallisto and Kraken exactly the same way as we ran them in the first microbial community found

280 in human-associated habitat, but without the filtration step to remove human reads.

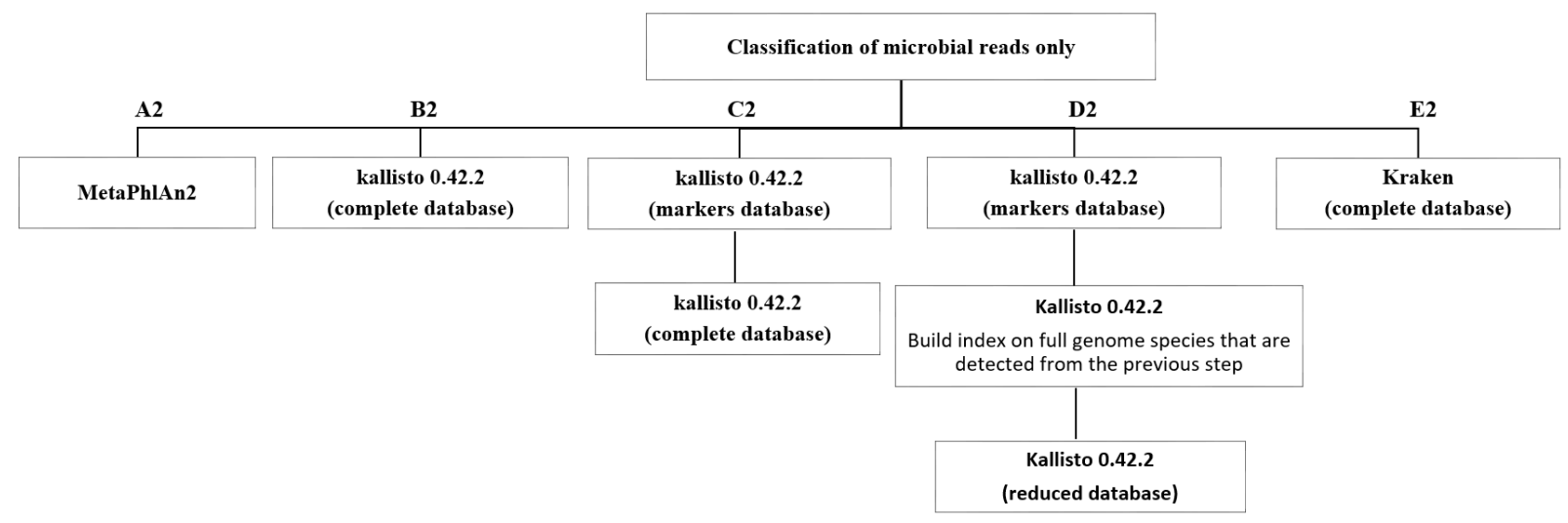

282 Figure 10: Flowchart of analysis pipelines for samples that consist of only microbial reads. Five 


\section{Reference genomes}

286 The hg19 reference genome was obtained from UCSC Genome Bioinformatics, University of

287 California, Santa Cruz [23]. Due to the very high memory requirement by kallisto, we included

288 only finished microbial sequences in our reference database based on available species-specific

289 markers from MetaPhlAn2 for the comparisons between five pipelines [6]. 3508 finished microbial

290 sequences that made up of 2616 microbial species, including phages, archaea, bacteria and viruses,

291 were obtained from the NCBI RefSeq database [19] and used in kallisto and Kraken pipelines. For

292 pipelines that involve the use of markers database, we included only markers that are found in the

2933508 finished microbial sequences. Quasi-markers and the set of markers that were excluded by

294 MetaPhlAn2 were not included in our marker database. A quasi-marker was identified as having

295 at least one external genome where it maps to. The same set of marker genes were used in our

296 MetaPhlAn2 pipeline. For the analysis of dataset from iMESS_Illumina simulated Illumina100

297 [20], we used all species-specific marker genes available at the species level from MetaPhlAn2 as

298 our first step of analysis. These markers are from 109985 microbial reference sequences (4868

299 bacterial, viral and fungal species). The subsequent step of building a reduced database (Figure 9

300 D1 and Figure 10 D2) uses sequences from 109985 fasta entries.

\section{Stool and saliva sequence data from the HPFS cohort}

302 The stool and saliva whole genome shotgun sequencing data from eight healthy HPFS male 303 participants were obtained from the Sequence Read Archive (accession number: PRJNA188481) $304[14]$.

\section{Quality Control}


To extract high quality reads, we filtered iMESSi-generated reads and the DNA sequencing reads from the HPFS cohort using prinseq v0.20.4 [24] with options -min_len 40, -trim_qual_left 10, trim_qual_right 10,-min_qual_mean 18.

\section{Evaluation of performance} requirement, and (vi) runtime.

\section{Statistical analysis}

For the analysis of metagenomic data from the HPFS cohort, we used estimated counts from $\log _{2}$ CPM.

\section{References}

1. Garza, D.R. and B.E. Dutilh, From cultured to uncultured genome sequences: metagenomics and modeling microbial ecosystems. Cell Mol Life Sci, 2015. 72(22): p. 4287-308.

2. Oulas, A., et al., Metagenomics: tools and insights for analyzing next-generation sequencing data derived from biodiversity studies. Bioinform Biol Insights, 2015. 9: p. 75-88.

3. Mande, S.S., M.H. Mohammed, and T.S. Ghosh, Classification of metagenomic sequences: methods and challenges. Brief Bioinform, 2012. 13(6): p. 669-81.

4. Sohn, M.B., et al., Accurate genome relative abundance estimation for closely related species in a metagenomic sample. BMC Bioinformatics, 2014. 15: p. 242.

5. Nguyen, N.P., et al., TIPP: taxonomic identification and phylogenetic profiling. Bioinformatics, 2014. 30(24): p. 3548-55. 
6. Truong, D.T., et al., MetaPhIAn2 for enhanced metagenomic taxonomic profiling. Nat Meth, 2015. 12(10): p. 902-903.

7. Liu, B., et al., Accurate and fast estimation of taxonomic profiles from metagenomic shotgun sequences. BMC Genomics, 2011. 12 Suppl 2: p. S4.

8. Segata, N., et al., Metagenomic microbial community profiling using unique clade-specific marker genes. Nat Methods, 2012. 9(8): p. 811-4.

9. Wood, D.E. and S.L. Salzberg, Kraken: ultrafast metagenomic sequence classification using exact alignments. Genome Biol, 2014. 15(3): p. R46.

10. Ounit, R., et al., CLARK: fast and accurate classification of metagenomic and genomic sequences using discriminative k-mers. BMC Genomics, 2015. 16: p. 236.

11. Lindgreen, S., K.L. Adair, and P.P. Gardner, An evaluation of the accuracy and speed of metagenome analysis tools. Sci Rep, 2016. 6: p. 19233.

12. Bray, N.L., et al., Near-optimal probabilistic RNA-seq quantification. Nat Biotechnol, 2016. 34(5): p. 525-7.

13. Schaeffer, L., et al. Pseudoalignment for metagenomic read assignment. ArXiv e-prints, 2015. 1510, 7371.

14. Franzosa, E.A., et al., Relating the metatranscriptome and metagenome of the human gut. Proc Natl Acad Sci U S A, 2014. 111(22): p. E2329-38.

15. Feehery, G.R., et al., $A$ method for selectively enriching microbial DNA from contaminating vertebrate host DNA. PLoS One, 2013. 8(10): p. e76096.

16. Neelakanta, G. and H. Sultana, The use of metagenomic approaches to analyze changes in microbial communities. Microbiol Insights, 2013. 6: p. 37-48.

17. Wu, G.D., et al., Linking long-term dietary patterns with gut microbial enterotypes. Science, 2011. 334(6052): p. 105-8.

18. Chen, H. and W. Jiang, Application of high-throughput sequencing in understanding human oral microbiome related with health and disease. Front Microbiol, 2014. 5: p. 508.

19. Tatusova, T., et al., RefSeq microbial genomes database: new representation and annotation strategy. Nucleic Acids Res, 2014. 42(Database issue): p. D553-9.

20. Mende, D.R., et al., Assessment of metagenomic assembly using simulated next generation sequencing data. PLoS One, 2012. 7(2): p. e31386.

21. Li, H., et al., The Sequence Alignment/Map format and SAMtools. Bioinformatics, 2009. 25(16): $\mathrm{p}$. 2078-9.

22. $\mathrm{Li}, \mathrm{H}$. Aligning sequence reads, clone sequences and assembly contigs with BWA-MEM. ArXiv eprints, 2013. 1303, 3997.

23. Kent, W.J., et al., The human genome browser at UCSC. Genome Res, 2002. 12(6): p. 996-1006.

24. Schmieder, R. and R. Edwards, Quality control and preprocessing of metagenomic datasets. Bioinformatics, 2011. 27(6): p. 863-4.

25. Robinson, M.D., D.J. McCarthy, and G.K. Smyth, edgeR: a Bioconductor package for differential expression analysis of digital gene expression data. Bioinformatics, 2010. 26(1): p. 139-40. 